\title{
Hypocholesterolaemic effects of a sodium bicarbonated mineral water in young subjects
}

\author{
M. P. Vaquero, A. M. Pérez-Granados, S. Navas-Carretero and S. Schoppen, \\ Instituto del Frío, ICTAN, Spanish National Research Council (CSIC), Madrid, Spain
}

The beneficial role of drinking a sodium bicarbonated mineral water on postprandial lipaemia and cardiovascular risk in healthy postmenopausal women has been described ${ }^{(1,2)}$. The present work aims to study the effect of drinking this mineral water on lipid metabolism in young men and women with moderate cardiovascular risk.

Eighteen young volunteers (ten women and eight men; 29 (SD 8) years) presenting with total cholesterol levels $>5.2 \mathrm{mmol} / \mathrm{l}$ and without any disease completed the study. The study consisted of two consecutive 8 -week intervention periods as described previously ${ }^{(1)}$. Subjects consumed daily 1 litre control mineral water (Font D'Or spring; Vichy Catalán SA, Barcelona, Spain) during the first period and 1 litre sodium bicarbonated mineral water (Vichy Catalán spring; Vichy Catalán SA) during the second period. Food intake, blood pressure, total cholesterol, LDL-cholesterol, HDL-cholesterol, apoB, TAG and glucose were determined. Data were analysed by repeated measures ANOVA.

\begin{tabular}{|c|c|c|c|c|c|}
\hline & \multicolumn{2}{|c|}{ Control water } & \multicolumn{2}{|c|}{ Bicarbonated water } & \multirow[b]{2}{*}{$P$} \\
\hline & Mean & SD & Mean & SD & \\
\hline Total cholesterol (mmol/l) & 5.78 & 0.73 & 5.42 & 0.67 & 0.012 \\
\hline HDL-cholesterol (mmol/l) & 1.51 & 0.31 & 1.56 & 0.33 & NS \\
\hline LDL-cholesterol (mmol/l) & 3.77 & 0.69 & 3.41 & 0.67 & 0.001 \\
\hline TAG $(\mathrm{mmol} / \mathrm{l})$ & 1.30 & 0.65 & 1.20 & 0.57 & NS \\
\hline Glucose (mmol/l) & 4.85 & 0.43 & 4.65 & 0.31 & NS \\
\hline ApoB $(g / l)$ & 1.35 & 0.41 & 1.06 & 0.21 & 0.017 \\
\hline Systolic blood pressure (mmHg) & 120 & 19 & 115 & 18 & 0.023 \\
\hline Diastolic blood pressure (mmHg) & 71 & 12 & 72 & 11 & NS \\
\hline
\end{tabular}

Nutrient intake did not vary between the water periods. Total cholesterol, LDL-cholesterol, apoB and systolic blood pressure significantly decreased after consumption of the sodium bicarbonated water. Differences between men and women in water response were not significant. In agreement with previous results ${ }^{(1,2)}$, consumption of the sodium bicarbonated mineral water reduced cardiovascular risk in young subjects who are moderately hypercholesterolaemic.

The study was supported by Vichy Catalán SA, Spain.

1. Schoppen S, Pérez-Granados AM, Carbajal A et al. (2004) J Nutr 134, 1058-1063.

2. Schoppen S, Pérez-Granados AM, Carbajal A et al. (2005) Br J Nutr 94, 582-587. 